\title{
Об оценках электронного сродства политипов карбида кремния и разрывов зон в гетеропереходах на их основе
}

\author{
(C) С.Ю. Давыдов
}

Физико-технический институт им. А.Ф. Иоффре Российской академии наук, 194021 Санкт-Петербург, Россия

E-mail: Sergei_Davydov@mail.ru

(Поступила в Редакцию 8 октября 2018 г.

В окончательной редакции 10 декабря 2018 г.

Принята к публикации 17 декабря 2018 г.)

\begin{abstract}
Обсуждаются две различные схемы оценок электронного сродства политипов $\mathrm{SiC}$ и их связь с результатами расчетов из первых принципов. Предложены простые поправки к правилам Шокли-Андерсона для построения зонных диаграмм гетеропереходов.
\end{abstract}

DOI: 10.21883/FTP.2019.05.47568.8995

Несмотря на более полувека интенсивного изучения, карбид кремния по-прежнему привлекает внимание исследователей как широкозонный материал с повышенной стойкостью к температурным, механическим и радиационным воздействиям [1-3]. В последнее десятилетие карбид кремния обрел новую область применения: было показано, что с помощью термодесорбции атомов кремния на поверхности $\mathrm{SiC}$ могут быть созданы слои графена [4]. Характерной особенностью карбида кремния является наличие большого числа политипов с кубической $(3 C)$, гексагональной $(\mathrm{N} H, \mathrm{~N}=2,4,6,8, \ldots)$ и ромбоэдрической $(\mathrm{N} R, \mathrm{~N}=15,21,27, \ldots)$ структурами. При этом различие ширин запрещенных зон $E_{g}$ наиболее часто используемых политипов $\mathrm{SiC}$ составляет величину $\sim 1$ эВ. Это дает возможность создавать гетеропереходы между химически одинаковыми компонентами контакта, избежав размывающей переход встречной диффузии приконтактных атомов и дислокаций несоответствия на интерфейсе.

Для составления энергетической диаграммы контакта, образованного политипами 1 и 2, необходимо знать ширины запрещенных зон $E_{g 1}, E_{g 2}$ и величины электронного сродства $\chi_{1}, \chi_{2}[5,6]$. Расчетам значений $E_{g}$ для политипов $\mathrm{SiC}$ посвящена обширная литература (см., например, [1,2,7-9]), чего нельзя сказать о расчетах $\chi$. Более того, в популярных справочниках $[10,11]$, где присутствует большой объем данных по политипам $\mathrm{SiC}$, об электронном сродстве даже не упоминается.

В качестве характеристик конкретных гетеропереходов удобно использовать разрывы зон проводимости и валентных зон на контакте, равные, в соответствии с моделью Шокли-Андерсона, соответственно

$$
\Delta E_{C}=\chi_{1}-\chi_{2}, \quad \Delta E_{V}=\phi_{2}-\phi_{1},
$$

где $\phi_{1,2}=\chi_{1,2}+E_{g 1,2}-$ энергии ионизации полупроводников 1 и 2 [5,12]. Для гетероконтактов политипов $\mathrm{SiC}$ некоторая разрозненная экспериментальная информация по величинам $\Delta E_{C}$ и $\Delta E_{V}$ имеется $[3,6]$, но какая-либо систематизация таких данных отсутствует.
Насколько известно автору, первая попытка оценки величин $\chi$ для политипов $\mathrm{SiC}$ была предпринята в работах $[13,14]$. Предполагалось, что

$$
\chi(D)=\chi_{3 C}-a D
$$

где $D=n_{\text {hex }} /\left(n_{\text {hex }}+n_{\text {cube }}\right)-$ степень гексагональности политипа [15] (см. таблицу, строка 1), $n_{\text {cube }}$ и $n_{\text {hex }}-$ числа занятых атомами кубических и гексагональных

Степень гексагональности $D$, ширина запрещенной зоны $E_{g}$

\begin{tabular}{|c|c|c|c|c|c|c|c|c|c|}
\hline № & Политип & $3 C$ & $8 H$ & $21 R$ & $6 H$ & $15 R$ & $27 R$ & $4 H$ & $2 H$ \\
\hline 1 & $D$ & 0 & 0.25 & 0.29 & 0.33 & 0.40 & 0.44 & 0.50 & 1 \\
\hline 2 & $E_{g}, \ni \mathrm{B}$ & 2.40 & 2.86 & 2.96 & 3.00 & 3.06 & 3.13 & 3.23 & 3.33 \\
\hline 3 & $\chi$, эВ & & & & & & & & \\
\hline & CBOA & 4.00 & 3.58 & 3.52 & 3.45 & 3.33 & 3.27 & 3.17 & 2.33 \\
\hline & $\begin{array}{l}\text { VBOA } \\
\text { [14], } \\
\text { теория }\end{array}$ & 4.00 & 3.57 & 3.48 & 3.44 & 3.39 & 3.33 & 3.24 & 3.20 \\
\hline 4 & $\begin{array}{c}\chi, \text { эВ } \\
{[16],} \\
\text { теория }\end{array}$ & 3.97 & 3.55 & 3.50 & 3.33 & 3.30 & 3.22 & 3.09 & 3.02 \\
\hline 5 & $\begin{array}{c}\chi, \text { эВ } \\
{[17],} \\
\text { эксперимент }\end{array}$ & 3.83 & & & 3.34 & & & 3.08 & \\
\hline 6 & $\begin{array}{c}\chi, \text { эВ } \\
{[18],} \\
\text { эксперимент }\end{array}$ & & & & 4.0 & & & $3.4-3.6$ & \\
\hline 7 & $\begin{array}{c}\chi, \text { эВ } \\
{[21],} \\
\text { теория }\end{array}$ & 3.83 & & & & & & 3.24 & \\
\hline 8 & $\begin{array}{c}\chi, \text { эВ } \\
{[22],} \\
\text { эксперимент }\end{array}$ & & & & & & & 3.47 & \\
\hline
\end{tabular}
и электронное сродство $\chi$ политипов карбида кремния*

Примечание. * Значения $D$ взяты из работы [15], $E_{g}$ - из работы [10], $\chi$ для политипа $3 C-\mathrm{SiC}-$ из работы [23]. 
позиций в решетке, $\chi_{3 C}=\chi(0)-$ электронное сродство кубического политипа $3 C$ - $\mathrm{SiC}, a-$ константа. Величина $a$ определялась по экспериментальным значениям $\Delta E_{C}$ для гетероперехода $3 C / 6 H-\mathrm{SiC}$ : согласно данным работы [14] имеем $\Delta E_{C}=a D=0.55$ эВ, так что $a=1.67$ эВ. Такая аппроксимация $\chi(D)$ обозначалась в работе [14] аббревиатурой СBОA (conduction band offset approximation). Аппроксимация VBOA (valence band offset approximation) строилась на основе соотношения $\Delta E_{V}=b D$ и теоретической оценки $\Delta E_{V}=0.13$ эВ для гетероперехода $3 C / 2 H$, так что $b=0.13$ эВ [14]. Результаты расчета $\chi$ представлены в таблице (строка 3 ).

Следующий шаг в этом направлении был сделан в работе [16]: значение электронного сродства вычислялось из соотношения $\chi=\bar{\phi}-E_{g}$, где под $\bar{\phi}$ понималось среднее экспериментальное значение энергии ионизации для политипов $3 C, 4 H$ и $6 H$, равное 6.35 эВ [17] (в работе [18] приводится значение $\bar{\phi}=6.9$ эВ), а величины $E_{g}$ рассчитывались из первых принципов. Результаты такой оценки $\chi$, приведенные в таблице (строка 4), хорошо согласуются со значениями, полученными в работе [14]. Дело в том, что предпринятая в работе [16] замена энергий ионизации различных политипов $\phi$ на среднее значение $\bar{\phi}$ эквивалентна условию $\Delta E_{V}=0$. Согласно оценкам [14], $b \ll 1$ эВ и, следовательно, $\Delta E_{V} \ll 1$ эВ, так что согласие результатов работ [14] и [16] не удивительно. То обстоятельство, что значения $\Delta E_{V}$ действительно малы для любой пары политипов $\mathrm{SiC}$, образующих гетеропереход, подтверждается расчетами $[19,20]$. Тогда, приняв для всех политипов $\phi=\bar{\phi}$, из (2) получим $E_{g}(D)=\bar{\phi}-\chi_{3 C}+a D$. Для сравнения с результатами работ [14] и [16] в таблицу включены данные экспериментальных и теоретических работ $[17,18,21-23]$ (строки 5-8).

Недавние расчеты из первых принципов [20,24,25] показали, что в полупроводниках имеются так называемые плавающие состояния (floating states), волновые функции которых не центрированы на узлах решетки, а делокализованы в пространстве межузельных каналов. По мнению авторов работ $[20,24,25]$, эти состояния определяют энергию дна зоны проводимости политипов $\mathrm{SiC}$ (т.е. $\chi$ ), тогда как за энергию потолка валентной зоны отвечают локализованные атомные состояния. Последнее обстоятельство приводит к малым различиям $\phi$, так как все политипы карбида кремния обладают идентичной структурой вплоть до третьей координационной сферы. С другой стороны, как следует из соотношения неопределенностей, средняя кинетическая энергия электронов в зоне проводимости имеет порядок $\hbar^{2} / 2 m_{e}(\Delta r)^{2}$, где $\Delta r-$ неопределенность координаты электрона, $m_{e}-$ эффективная масса электрона, $\hbar-$ приведенная постоянная Планка. Так как по данным работы [20] значения $\Delta r$ в гексагональных политипах $\mathrm{SiC}$ меньше, чем в кубическом политипе, получаем неравенства $E_{g}(3 C)<E_{g}(\mathrm{~N} H)$ и $\chi(3 C)>\chi(\mathrm{N} H)$.

Соотношения (1) модели Шокли-Андерсона не учитывают взаимовлияния контактирующих полупроводни- ков на их электронные спектры, что может приводить к значимым ошибкам при определении разрывов зон. Здесь имеется известная параллель с теорией адсорбции, где для оценки направления перехода заряда между адатомом и подложкой используют соотношение работы выхода подложки и энергии ионизации атома, а гибридизационный сдвиг квазиуровня адатома игнорируют [26].

Для оценки взаимовлияния контактирующих политипов вместо характеристик $\chi_{1(2)}$ и $\phi_{1(2)}$ введем

$$
\tilde{\chi}_{1(2)}=\chi_{1(2)}+\Delta \chi_{1(2)}, \quad \tilde{\phi}_{1(2)}=\phi_{1(2)}+\Delta \phi_{1(2)},
$$

где поправки $\Delta \chi_{1(2)}$ и $\Delta \phi_{1(2)}$ учитывают влияние политипа 2(1) на значения $\chi_{1(2)}$ и $\phi_{1(2)}$ политипа $1(2)$. В приближении линейного отклика представим $\Delta \chi_{1(2)}$ и $\Delta \phi_{1(2)}$ в виде

$$
\Delta \chi_{1(2)}=\Lambda_{1(2)}\left(\chi_{1(2)}\right), \quad \Delta \phi_{1(2)}=\Lambda_{1(2)}\left(\phi_{1(2)}\right),
$$

где

$$
\Lambda_{1(2)}(\varepsilon)=V^{2} \int_{-\infty}^{\infty} \frac{\rho_{2(1)}\left(\varepsilon^{\prime}\right) d \varepsilon^{\prime}}{\varepsilon^{\prime}-\varepsilon}
$$

$\varepsilon-$ энергия, $V-$ матричный элемент взаимодействия политипов 1 и $2, \rho_{1(2)}(\varepsilon)-$ энергетическая плотность состояний политипов 1 (2). Выражения (3)-(5) написаны по аналогии с моделями Андерсона-Ньюнса и Халдейна-Андерсона [27]. Для плотностей состояний политипов $4 H$ и $6 H$ примем модель из работы [28], использованную нами ранее в работе [29]. В упрощенном варианте [30] можно записать

$$
\rho_{1(2)}(\varepsilon)=A_{1(2)} \begin{cases}\sqrt{\varepsilon-E_{g 1(2)} / 2}, & \varepsilon>E_{g 1(2)} / 2, \\ \sqrt{-\varepsilon-E_{g 1(2)} / 2}, & \varepsilon<-E_{g 1(2)} / 2, \\ 0, & |\varepsilon| \leq E_{g 1(2)} / 2\end{cases}
$$

где $A-$ коэффициент $[28,29]$. Так как за нуль энергии в (6) принято положение центров запрещенных зон, $\phi_{1(2)}$ переходит в $-E_{g 1(2)} / 2$, а $\chi_{1(2)}-$ в $E_{g 1(2)} / 2$. Подставляя (6) в (5), получаем

$$
\Lambda_{1(2)}(\varepsilon)=A_{2(1)} V^{2} \begin{cases}F_{-}^{2(1)}(\varepsilon), & \varepsilon<-E_{g 2(1)} / 2, \\ F_{-}^{2(1)}(\varepsilon)-F_{+}^{2(1)}(\varepsilon), & |\varepsilon| \leq E_{g 2(1)} / 2, \\ -F_{+}^{2(1)}(\varepsilon), & \varepsilon>E_{g 2(1)} / 2,\end{cases}
$$

где $F_{ \pm}^{2(1)}(\varepsilon)=\pi \sqrt{ \pm \varepsilon+E_{g 2(1)} / 2}$. В таком упрощенном варианте мы свели различия политипов к разнице значений $E_{g}$ и $A$. Подчеркнем, что здесь, как и в $[27,28]$, мы для простоты не делаем различия между коэффициентами $A_{C}$ и $A_{V}$ для проводящей и валентной зон [28], полагая $A_{C}=A_{V}=A$.

Будем считать, что $E_{g 2}>E_{g 1}, \chi_{2}<\chi_{1}, \phi_{1}=\phi_{2}=\bar{\phi}$, и рассмотрим влияние широкозонного компонента гетероперехода на узкозонный. Примем за нуль энергию центра запрещенной зоны $E_{g 2}$, т. е. положим $\chi_{2}+E_{g 2} / 2=0$. 
При этом

$\chi_{2}=E_{g 2} / 2, \quad \phi_{2}=-E_{g 2} / 2, \quad \chi_{1}^{\prime}=E_{g 2}-\chi_{1}, \quad \phi_{1}^{\prime}=-E_{g 2} / 2$.

Получим

$$
\Delta \chi_{1}=-\pi A_{2} V^{3}\left(\sqrt{3 E_{g 2} / 2-\chi_{1}}-\sqrt{\chi_{1}-E_{g 2} / 2}\right)
$$

и

$$
\Delta \phi_{1}=-\pi A_{2} V^{2} \sqrt{E_{g 2}},
$$

где мы положили для простоты $\phi_{i}=\phi_{2}=\bar{\phi}$. Поменяв индексы 1 на индексы 2 и наоборот, получим поправки $\Delta \chi_{2}$ и $\Delta \phi_{2}$, описывающие результат воздействия узкозонного компонента на широкозонный.

По оценкам работы [30], для политипа $6 H$ $A \approx 0.2$ эВ $^{-3.2}$; тот же порядок множителя $A$ характерен и для других политипов (см., например, [26]). Таким образом, имеем $\pi A \sqrt{E_{g}} \sim 1$ э $\mathrm{B}^{-1}$. Сложнее оценить матричный элемент $V[26,27]$. Однако если даже предположить, что $V$ не превосходит 1 эВ, сдвиги $\Delta \chi_{1}$ и $\Delta \phi_{1}$ будут иметь порядок 1 эВ. Здесь следует, однако, учесть, что сдвиги $\Delta \chi_{1,2}$ и $\Delta \phi_{1,2}$ имеют одинаковые знаки и порядок величины, так что значения разрывов зон $\Delta E_{C}$ и $\Delta E_{V}$ изменяются на величину, существенно меньшую, чем $\left|\Delta \chi_{1,2}\right|$ и $\left|\Delta \phi_{1,2}\right|$.

Таким образом, сделанные нами оценки показали, вопервых, что два различных подхода к оценкам электронного сродства ([14] и [16]) дали почти идентичные результаты. Во-вторых, проведенный нами анализ взаимовлияния компонентов гетероконтакта продемонстрировал, что привлекательную своей простотой схему Шокли-Андерсона можно использовать для объемных образцов политипов $\mathrm{SiC}$, хотя и с известной осторожностью. Отметим, что сходная задача о влиянии подложки на электронную структуру графеноподобных соединений (ГПС), адсорбированных на полупроводниковой подложке, рассматривалась нами в работе [31], Было показано, что в отличие от рассмотренного здесь случая учет взаимодействия ГПС - подложка может качественно изменить зонную диаграмму контакта, построенную по схеме Шокли-Андерсона. В отличие от объемных полупроводников, однако, для ГПС теоретические значения электронного сродства известны $[32,33]$.

\section{Список литературы}

[1] Silicon Carbide: recent major advances, ed. by W.J. Choyke, H. Matsunami, G. Pensl (Berlin-Heidelberg, Springer, 2004). E-address: http://www.springer.de

[2] Advances in Silicon Carbide. Processing and Applications, ed. by S.E. Saddow, A. Agarwal (Boston-London, Artech House, 2004). E-address: www.artechhouse.com

[3] A.A. Lebedev. Semicond. Sci. Technol., 21, R17 (2006).

[4] H. Hibino, H. Kagoshima, M. Nagase. J. Phys. D: Appl. Phys., 42, 374005 (2010).

[5] Ф. Бехштедт, Р. Эндерлейн. Поверхности и границы раздела полупроводников (М., Мир, 1990).

[6] A. Fissel. Phys. Reports, 379, 149 (2003).
[7] W.R.L. Lambrecht, S. Limpijumnong, S.N. Rashkeev, B. Segall. Phys. Status Solidi B, 202, 5 (1997).

[8] F. Bechstedt, P. Kackell, A. Zywietz, K. Karch, B. Adolph, K. Tenelsen, J. Furthmüller. Phys. Status Solid B, 202, 35 (1997).

[9] G. Wellenhofer, U. Rossler. Phys. Status Solidi B, 202, 107 (1997).

[10] В.И. Гавриленко, А.М. Грехов, Д.В. Корбутяк, В.Г. Литовченко. Оптические свойства полупроводников. Справочник (Киев, Наук. думка, 1987).

[11] M. Shur, S. Rumyantsev, M. Levinshtein. Properties of advanced semiconductor matterials. GaN, AlN, InN, BN, SiC (N.Y., Wiley, 2001).

[12] E.T. Yu, J.O. McCaldin, T.S. McGill. Sol. St. Phys., 46, 1 (1992).

[13] С.Ю. Давыдов, А.А. Лебедев, О.В. Посредник. ФТП, 39, $1440(2005)$.

[14] С.Ю. Давыдов. ФТП, 41, 718 (2007).

[15] Н.Д. Сорокин, Ю.М. Таиров, В.Ф. Цветков, М.А. Чернов. Кристаллография, 28, 910 (1983).

[16] V.N. Brudny, A.V. Kosobutsky. Superlat. Microstruct., 111, 499 (2017).

[17] J. Kuriplach, M. Sob, G. Brauer, W. Anwand, E.-M. Nicht, P.G. Coleman, N. Wagner. Phys. Rev. B, 59, 1948 (1999).

[18] M. Wiets, M. Weinelt, T. Fauster. Phys. Rev. B, 68, 125321 (2003).

[19] W. van Haeringen, P.A. Bobbert, W.H. Backes. Phys. Status Solidi B, 202, 63 (1997).

[20] Y. Matsushita, S. Furuya, A. Oshiyama. J. Phys. Soc. Jpn, 83, 094713 (2014).

[21] A. Arvanitopoulos, N. Lophitis, S. Perkins, K.N. Gyftrakis, G. Belanche, M. Guadas, M. Antoniou. IEEE Int. Symp. on Diagnostics for Electric Machines, Power Electronics and Drives (2017).

E-address: http://dx.doi.org/10.1109/DEMPED.2017.8062411

[22] N. Fujimura, A. Ohta, K. Makihara, S. Miyazaki. Jpn. J. Appl. Phys., 55, 08PC06 (2016).

[23] M. Rejhon, J. Franc, V. Dedic, P. Hlidek, J. Kunc. arXiv: 1712.02563

[24] Y. Matsushita, S. Furuya, A. Oshiyama. Phys. Rev. Lett., 108, 246404 (2012).

[25] Y. Sugihara, K. Uchida, A. Oshiyama. J. Phys. Soc. Jpn, 84, 084709 (2015).

[26] С.Ю. Давыдов. Теория адсорбиии: метод модельных гамильтонианов (СПб., Изд-во СПбГЭТУ „ЛЭТИ“, 2013). twirpx.com/file/1596114/

[27] С.Ю. Давыдов, С.В. Трошин. ФТТ, 49, 1508 (2007).

[28] C. Persson, U. Lindefelt. Mater. Sci. Forum, 264-268, 275 (1998).

[29] С.Ю. Давыдов. ЖТФ, 84 (4), 155 (2014).

[30] С.Ю. Давыдов. ФТП, 48, 49 (2014).

[31] С.Ю. Давыдов. ФТТ, 58, 1182 (2016).

[32] C.-J. Tong, H. Zhang, Y.-N. Zhang, H. Liu, L.-M. Liu. J. Mater. Chem. A, 2, 17971 (2014).

[33] И.В. Антонова. ФТП, 50, 67 (2016).

Редактор А.Н. Смирнов

Физика и техника полупроводников, 2019, том 53, вып. 5 
On the estimates of the electron affinity

for silicon carbide polytypes and band offsets in heterojunctions on their bases

S.Yu. Davydov

loffe Institute,

194021 St. Petersburg, Russia

Abstract Two different schemes for estimates of SiC polytypes and their relation with the ab initio calculated results are discussed, Simple corrections to the Shockley-Anderson rules for the construction of heterojunction band diagram are proposed. 